\title{
Contribution of Finite Element Analyses to Crack-Like Flaw Assessments in a Gas Pipeline
}

\author{
Thibault Jacquemin ${ }^{\mathrm{a}, \mathrm{b}, ~ *}$, Mehmet E. Kartal ${ }^{\mathrm{b}}$, Gonzague Du Suauc, Stéphane Hertz- \\ Clémens ${ }^{\mathrm{C}}$ \\ ${ }^{a}$ Wood Group, Galway, Ireland \\ ${ }^{b}$ School of Engineering, University of Aberdeen, King's College, Aberdeen AB24 3UE, United Kingdom \\ ${ }^{c}$ Wood Group, Paris, France
}

\begin{abstract}
When defects reach a critical size, failure occurs in engineering components. The criticality assessment of defects is hence a key aspect of the structural integrity of gas pipelines in service. Fitness-For-Service (FFS) assessments are generally employed for evaluating the criticality of a crack-like flaw in structures using simplified assumptions in relation to geometry and material properties. Whilst the errors resulting from these modelling simplifications prove acceptable in many cases, there are situations where it will be necessary to take into account the nonlinearities in geometry and/or material behaviour. This can be either to avoid excess conservatism or, on the contrary, to ensure the results are safe. In such cases it becomes essential to develop a finite element model of the structure to account for such real-engineering complexity. Welding, the most prevalent technique to join pipe, often brings about a misalignment between two pipes and hence complex crack shape is formed. The aim of this study is to develop an elastoplastic finite element model of a gas pipeline possessing a crack in a misaligned weld. The remaining life of the pipeline is determined using a Failure Assessment Diagram (FAD) and the Paris law. The results obtained from the finite element method to determine the stress intensity factors are compared to results derived using the API-579 for stress intensity factors calculations.
\end{abstract}

Keywords: Gas Pipeline, Hi-Lo Misalignment, Crack, Fracture, Steel, API-579;

\section{Introduction}

Pipeline plays a significant role in the energy sector and whose structural integrity is a key issue for the safe production. In-service pipes are generally subjected to complex loading regimes. While the internal pressure is the primary source of loading, additional loading due to ground movements such as landslide can compromise the integrity of a pipeline and result in fatalities or damage to the environment.

During the life of a gas pipeline, non-destructive testing is performed on a regular basis to determine the presence and size of defects. Stress cycles (mostly due to the cyclic internal pressure loading of the pipeline) can lead to the progression of the cracks and can hence accelerate failure. In order to ensure the integrity of the pipeline and to avoid catastrophic failure, an assessment needs to be performed each time a defect is found. If the defect is deemed "unacceptable", the affected portion of the pipeline is repaired or replaced.
In steel welded structures, crack-like flaws are of particular importance as the welding process tends to induce such defects. The API 579-1/ASME FFS-1 "Fitness-For-Service" (API-579) [1] and the BS-7910 "Guide to methods for assessing the acceptability of flaws in metallic structures" [2] are the two standards typically used in the industry to assess crack-like flaw in a steel structure. In both standards, the Failure Assessment Diagram (FAD) approach is used [1], [2]. The assessment point corresponding to the analysed defect under the applied loading is placed on the FAD using semi-analytical formulae from the standards. The position of this point on the diagram allows determining of the acceptability of a crack and estimating the number of stress cycles leading the defect to an unacceptable region.

However, these standards are commonly appropriate in an elastic material and in general deemed conservative. In the case of having more complicated cases such as material non-linearity, a finite element model has to be developed to predict failure in the pipeline. 
Zhang et al. analysed the influence of the crack dimensions on the observed CTOD in a number of papers [3], [4], [5] using load-based and strain-based loadings. However, the results obtained from finite element analyses were not compared against the standards.

A fracture analysis of a thin-walled pipe was presented in [6]. The considered pipeline had a diameter of $914 \mathrm{~mm}$ and was loaded with internal pressure. The results presented in this work compared J-integrals calculated from experiments to the results derived with the FE method. FE results are derived from a "shell" finite element model. The behaviour of the crack in the thickness of the pipeline was consequently not accurately described and the case of a misalignment was not treated.

Chong et al. [7] and Yi et al. [8] analysed the fracture capacity of pipelines for surface and embedded crack respectively. FE results have been compared to analytical results derived from the BS-7910. The calculated J-Integrals or CTOD from the FE analysis have been shown to be in general smaller than the ones given by the BS-7910. No pipeline misalignment was considered in these studies and the gain in terms of life was not assessed.

The influence of a misalignment at the weld location was studied in [9] for the case of a plate configuration. In this paper, results were compared to results calculated from the API-579.The influence of a strain based assessment in the study of pipeline failure and fracture was covered in the work of [10] and [11]. Guidelines for a strain based design of pipelines for both onshore and offshore applications was provided in [12].

The case of an onshore gas pipeline having a Hi-Lo misalignment has been considered in this study as little references have been found for this common type of defect. Finite element models have been developed in order to determine the severity of crack-like flaws of various dimensions.

Two and three-dimensional calculations have been performed using the finite element solver ABAQUS [13], [14]. Results derived from non-linear finite element models have been compared to results derived from the API-579 standard.

\begin{tabular}{|ll|}
\hline \multicolumn{2}{|l|}{ Abbreviations } \\
\hline API & American Petroleum Institute \\
BS & British Standard \\
CTOD & Crack Tip Opening Displacement \\
EPFM & Elastic Plastic Fracture Mechanics \\
FE & Finite Element \\
FEA & Finite Element Analysis \\
FEM & Finite Element Method \\
FoS & Factor of Safety \\
HAZ & Heat Affected Zone \\
LEFM & Linear Elastic Facture Mechanics \\
SIF & Stress Intensity Factor \\
SSY & Small Scale Yielding \\
WG & Wood Group \\
\hline
\end{tabular}

\section{Materials and geometry}

The pipeline analysed is an onshore gas pipeline having a High-Low (Hi-Lo) misalignment. A Hi-Lo misalignment is a linear misalignment observed when one of the welded sections is higher than the other. Such defect is commonly observed in onshore gas pipelines due to their large diameter and relatively small wall thickness. The allowable misalignment during the welding operation needs to be determined. Fig. 1 presents the shape of a circumferential weld of the considered pipeline. It should be noted that a weld is never totally "defect" free. Hence, allowable welding defect size is a key parameter in a pipeline design, which can be provided in the code.

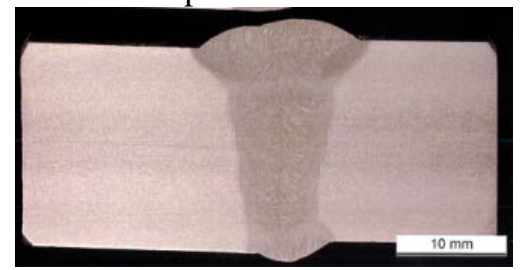

Fig. 1 Observed Welds at Hi-Lo Misalignment Location

The main dimensions and the material properties of the considered gas pipeline are respectively presented in Table 1 and Table 2. 
Table 1. Pipeline Dimensions

\begin{tabular}{lccc}
\hline \multicolumn{1}{c}{ Parameter } & Symbol & Value & Unit \\
\hline Outer Diameter & $\mathrm{D}$ & 1219.2 & $\mathrm{~mm}$ \\
\hline Thickness & $\mathrm{t}$ & 13 & $\mathrm{~mm}$ \\
\hline $\begin{array}{l}\text { Hi-Lo Misa- } \\
\text { lignment }\end{array}$ & $\mathrm{e}$ & 3.9 & $\mathrm{~mm}$ \\
\hline
\end{tabular}

Table 2. Material Properties ${ }^{(1)}$

\begin{tabular}{lccc}
\hline \multicolumn{1}{c}{ Parameter } & Symbol & Value & Unit \\
\hline Young Modulus & $\mathrm{E}$ & 200,000 & $\mathrm{MPa}$ \\
\hline Poisson Ratio & $v$ & 0.3 & - \\
\hline Yield Strength & $\sigma_{\mathrm{Y}}$ & 485 & $\mathrm{MPa}$ \\
\hline $\begin{array}{l}\text { Ultimate Tensile } \\
\text { Strength }\end{array}$ & $\mathrm{UTS}$ & 570 & $\mathrm{MPa}$ \\
\hline $\begin{array}{l}\text { Maximum } \\
\text { deformation }\end{array}$ & $\varepsilon_{\mathrm{Max}}$ & 0.18 & - \\
\hline $\begin{array}{l}\text { Stress / Strain } \\
\text { Relation }\end{array}$ & - & $\begin{array}{c}\text { Ramberg } \\
\text { Osgood }\end{array}$ & - \\
\hline $\begin{array}{l}\text { Fracture } \\
\text { Toughness }\end{array}$ & $\mathrm{K}_{\mathrm{IC}}$ & 131.8 & $\mathrm{MPa} \cdot \sqrt{\mathrm{m}}$ \\
\hline $\begin{array}{l}\text { (1) } \\
\text { The material properties of a typical onshore gas pipeline have been } \\
\text { selected following the requirement of the gas pipeline joint venture } \\
\text { C4Gas [15] and these are typical values for the material considered } \\
\text { in this study [16] }\end{array}$ & &
\end{tabular}

The Ramberg Osgood stress-strain relation of the considered pipe material is presented Fig. 2 below.

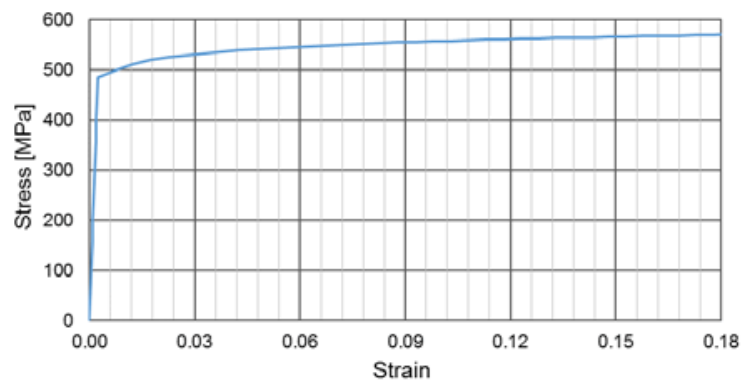

Fig. 2 Ramberg Osgood Stress-Strain Curve

\section{Analytical crack-tip stress fields in 2D media}

In the case of a crack in the circumferential direction of a pipeline under internal pressure and subject to tension and/or bending, the fracture Mode I (opening mode) is dominant.

A crack can be analysed under different assumptions depending on loading, material and the level of precision required. The standards used for crack-like flaw assessments are based on an elastic analysis of the stress field around the crack tip. This approach is known as the Linear Elastic Fracture Mechanics (LEFM). The stress field around the crack tip is a combination of the contribution from three fracture modes.

$$
\sigma_{i j}(r, \theta)=\frac{1}{\sqrt{2 \pi r}}\left[K_{I} f_{i j}^{I}(\theta)+K_{I I} f_{i j}^{I I}(\theta)+K_{I I I} f_{i j}^{I I I}(\theta)\right]
$$

where:

- $\quad r$ is the distance from the crack tip;

- $\theta$ is the angle from the crack direction;

- $\mathrm{K}_{\mathrm{I}}, \mathrm{K}_{\mathrm{II}}$ and $\mathrm{K}_{\mathrm{III}}$ are the stress intensity factors associated respectively to the modes I, II and III;

- $f_{i j}^{I}(\theta), f_{i j}^{I I}(\theta)$ and $f_{i j}^{I I I}(\theta)$ are three angular functions.

From this equation, it can be noticed that at the crack tip $(r=0)$ the stress is infinite. The stress increases in as a function of $\frac{1}{\sqrt{r}}$ when $r$ tends to zero.

\subsection{Stress Intensity Factors and J-Integrals}

The stress intensity factors $\left(\mathrm{K}_{\mathrm{I}}, \mathrm{K}_{\mathrm{II}}\right.$ and $\mathrm{K}_{\mathrm{III}}$ in Equation (1)) are the values characterizing the influence of loading or deformation on the stress field around the crack tip. These values are used to determine if a crack is likely to lead to fracture.

The stress intensity factors are used in LEFM and are the parameters calculated when using the standards (e.g. API-579).

Similarly, to the Stress Intensity Factor, the "JIntegral" is a parameter characterizing the state of a crack. This factor is often used as it can be calculated both for elastic and plastic assessments and its value is a function of the stain energy release and the created surface. The J-Integral can also be readily measured from experiments and FE models.

In two and three-dimensional domains, the JIntegral is calculated in a plane orthogonal to the crack front based on the equations:

$$
\begin{gathered}
J=\oint_{\Gamma}\left(\mathrm{W} * \mathrm{dx}_{2}-\mathrm{t}_{\mathrm{i}} \frac{\partial \mathrm{u}_{\mathrm{i}}}{\partial \mathrm{x}_{1}} \mathrm{ds}\right) \\
\mathrm{W}=\int_{0}^{\epsilon} \sigma: \mathrm{d} \boldsymbol{\epsilon}
\end{gathered}
$$

where:

- $\mathrm{x}_{1}, \mathrm{x}_{2}$ are the coordinate directions in a plane orthogonal to the crack front

- $\mathrm{W}\left(\mathrm{x}_{1}, \mathrm{x}_{2}\right)$ is the strain energy release

- $\quad t$ is the surface traction 
- $\boldsymbol{\sigma}$ is the Cauchy stress tensor

- $\quad \mathbf{u}$ is the displacement vector

The Stress Intensity Factors and the J-Integral can be related [14] as

$$
\mathrm{J}=\frac{1}{8 \pi} \mathbf{K}^{\mathrm{T}} \cdot \mathbf{B}^{-1} \cdot \mathbf{K}
$$

where:

- $\mathbf{K}=\left[\mathrm{K}_{\mathrm{I}}, \mathrm{K}_{\mathrm{II}}, \mathrm{K}_{\mathrm{III}}\right]$

- $\mathbf{B}$ is the pre-logarithmic energy factor matrix

In ABAQUS finite element software the stress intensity factors are derived from the J-Integral. Hence results in terms of J-Integral has been presented in this study.

For cracks under mode I loading in linear elastic materials, the relation between the J-Integral and the stress intensity factor becomes [1]:

$$
\mathrm{J}=\mathrm{K}_{\mathrm{I}}^{2} *\left(\frac{1-v^{2}}{\mathrm{E}}\right)
$$

This formula is valid for two-dimension models subject to plane strain loading and three-dimension models.

\subsection{J-Integral Contours}

As presented in Equation (2), the J-Integral is calculated based on a closed - one dimension - contour. It has been proven by Rice in 1968 [17] that the calculated integral is independent from the path of the contour.

In order to improve the accuracy of the calculated J-Integral, it is recommended to calculate a number of integrals and to consider the average value from these. Only the contours for which the J-Integral from one contour to the next one is sufficiently "stable" shall be considered.

For elastic-plastic FE analyses, the stability of the J-Integral contours is achieved relatively far from the crack tip compared to models with linear elastic materials.

Fig. 3 shows the path of the contour selected in ABAQUS. The portion $\Gamma$ of the contour is taken as close as possible to the crack tip.

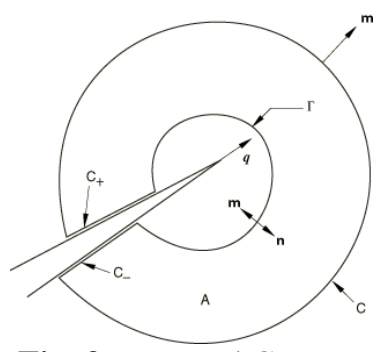

Fig. 3 J-Integral Contour

\section{FE Crack Modelling Approaches}

\subsection{Sharp Crack Tip}

The LEFM theory is based on the assumption of a sharp crack tip leading to a $r^{-0.5}$ singularity in the stress field. For an elastic-plastic analysis, the ABAQUS documentation recommends to accentuate the stress increase near the crack tip by introducing a $r^{-1}$ singularity.

In order to model these two types of singularity in a finite element model, the shape functions of the quadratic elements surrounding the crack tip are modified. The elements at the crack tip location are collapsed as presented Fig. 4. In order to model a $r^{-0.5}$ singularity, the mid-side nodes are moved to $1 / 4^{\text {th }}$ of the edge toward the tip. The $r^{-1}$ singularity is achieved by leaving the mid-side nodes at their original location (centre of the element edge).

The particularity of the quadratic bricks (C3D20 in ABAQUS) is that collapsing one side of the element leads to a non-constant stress field in the element. To achieve a constant $r^{-0.5}$ stress singularity across the degenerated elements, the edge plane nodes are displaced together as presented Fig. 4. 


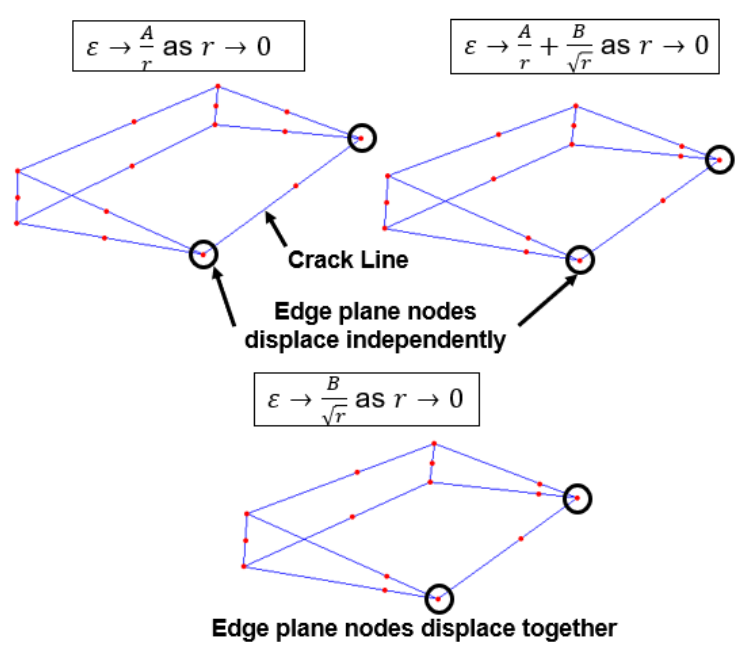

Fig. 4 Degenerated C3D20 Elements [13], [14]

It should also be noted that J-Integral contours comprising the mid-side nodes generally lead to lower calculated J-Integrals as fewer nodes are perturbed by the deformation. These contours will not be considered in the J-Integral calculation.

\subsection{Blunt Crack Tip}

Observations and experiments show that in reality the crack tip is not perfectly sharp. Under loading the crack tip tends to blunt as presented Fig. 5 . The blunt radius and the size of the elements modelling the tip should be chosen carefully based on the size of the plastic zone. It is recommended to select a blunt radius of $10^{-3}$ the size of the plastic zone and to choose elements of about $1 / 10^{\text {th }}$ of the blunt radius at the crack tip. This allows the deformed shape of the crack tip to no longer depend on the original geometry [14].

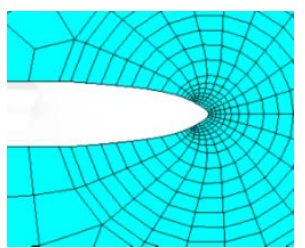

Fig. 5 Blunt Crack Tip FE Model [13]

\section{FEM vs Newman Raju Formulation}

In order to validate the use of ABAQUS to derive J-Integrals and to assess the impact of the mesh density on the calculated results, a 3D model has been analysed and compared to an analytical solution. The results obtained from the FEM are compared against the Newman-Raju formulation. Comparison is made for the case of a semi-elliptical crack in a plate under tension as this configuration is related to a crack observed in a gas pipeline.

\subsection{The Newman-Raju Formulation}

The Newman-Raju formula is a widely used and recognised formula for calculating crack stress intensity factors in plates. It is valid for linear elastic materials. The Newman-Raju formulation for semielliptical cracks in plates is presented in [17]. The stress intensity calculation is presented in Equation (6) as a function of the ellipse parametric angle $\phi$ presented in Fig. 6.

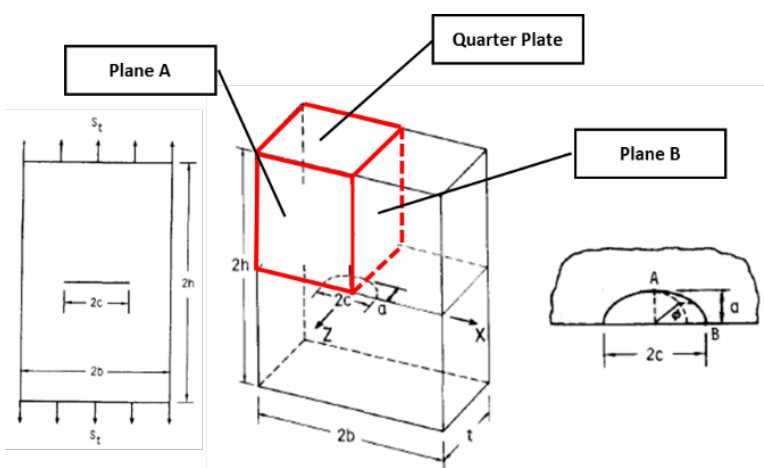

Fig. 6 Newman-Raju Surface Crack Parameters and Loading Condition [17]

This formulation only applies to surface cracks with $0<\mathrm{a} / \mathrm{c} \leq 1.0,0 \leq \mathrm{a} / \mathrm{t}<1.0$ and $\mathrm{c} / \mathrm{b}<0.5$. The parameters $a, b, c$ and $t$ are presented in Fig. 6 .

$$
\mathrm{K}_{\mathrm{I}}(\phi)=\left(\mathrm{S}_{\mathrm{t}}+\mathrm{HS}_{\mathrm{b}}\right) \sqrt{\pi \frac{\mathrm{a}}{\mathrm{Q}}} * \mathrm{~F}\left(\frac{\mathrm{a}}{\mathrm{t}}, \frac{\mathrm{a}}{\mathrm{c}}, \frac{\mathrm{c}}{\mathrm{b}}, \phi\right)
$$

where:

- $\quad S_{t}$ is the surface traction applied on the plate

- $\quad S_{b}$ is the bending moment that tends to open the crack

FE models and testing allowed determining the parameters of an empirical law for a range of crack dimensions. More details about the empirical functions $H, Q$ and $F$ can be found in [17].

\subsection{FE Model and Results}

Only the section highlighted in red in Fig. 6 has been modelled in ABAQUS due to the symmetries of 
the plate. Five models have been considered for various mesh densities and modelling approaches. The results given from a full one part and fully compatible model have been compared to a model where the ABAQUS “Tie Constraint" has been used.

Instead of modelling the plate considered by using a single part meshed with compatible elements, the plate can be split into several parts. The contact between two adjacent parts is defined by boundary conditions where all degrees of freedom are constrained (Tie Constraint) in order to re-create the integrity of the structure. This method significantly reduces the number of elements in the model and consequently allows having a finer mesh at the crack tip location. An overview of both models for the fine mesh density is presented in Fig. 7. It can be noticed from this figure that the stress field obtained from both modelling approaches is similar.

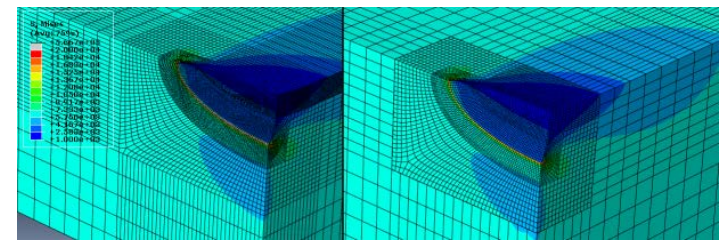

a)

b)

Fig. 7 Fine Mesh ABAQUS Models: (a) One-part Model (b) ABAQUS Tie Constraint Model

Fig. 8 shows the calculated J-Integral values obtained from various FE results and the Newman-Raju theoretical formulation along the crack front.

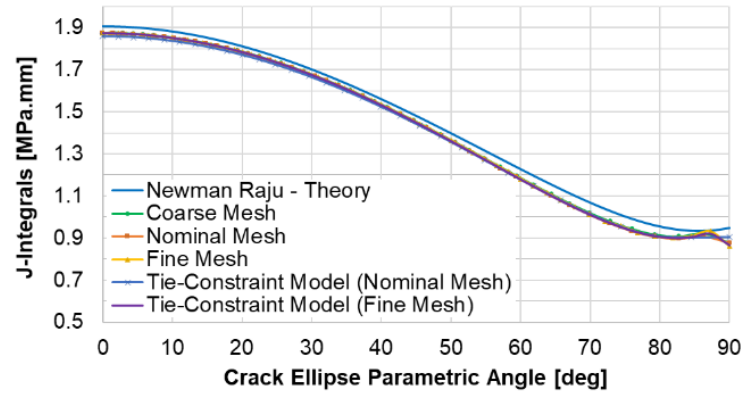

Fig. 8 Newman Raju vs FEA J-Integral Comparison

As can be seen from the figure, when a sharp crack tip modelling approach is considered, the impact of a mesh density around the crack tip on the calculated JIntegral is not significant. Furthermore, the use of a Tie Constraint does not really impact the results.

The Newman-Raju results are larger than the results calculated by the FE analysis. The average relative difference along the crack tip is $1.96 \%$. These results give confidence in the ability of an ABAQUS FE model to reproduce accurate results from a widely validated analytical law. The Newman Raju formula is deemed to be $\pm 5 \%$ accurate [17]; this can explain the reason for a small difference between two different approaches.

The use of the Tie Constraint allows reducing the complexity of the mesh and also reduces the element distortion. However, even if the number of elements is reduced, similar computation times are observed due to the additional contact iterations required.

\section{Axisymmetric Pipeline Analysis}

The preliminary study presented in Section 5 allowed gaining confidence in the results calculated from 3D FE models using ABAQUS. The purpose of the study presented in this section is to assess the criticality of a crack in a more complex and realistic geometry. An analysis of an axisymmetric model of the pipeline introduced Section 2 is presented in this section. A linear elastic material is considered in this section. Only internal pressure loading, corresponding to the maximum service pressure of the pipeline, is considered. End cap force has not been considered in the model. An overview of the considered model is presented in Fig. 9.

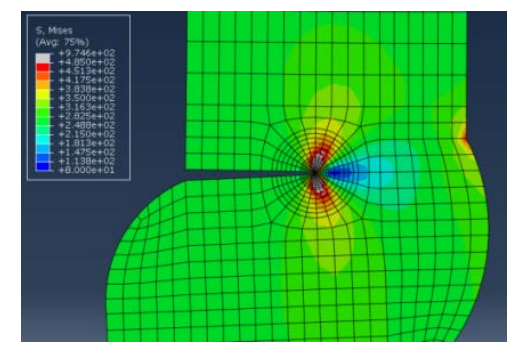

Fig. 9 Axisymmetric Pipeline Model $-\mathrm{a}=6 \mathrm{~mm}$

The results have been compared in terms of $\mathrm{J}$ Integral. Two crack tip modelling methods have been considered: a sharp and a blunt crack tip. The results calculated by the FEM have been compared to those derived from the API-579 [1].

\subsection{Stress Field Across Thickness}

To account for the Hi-Lo weld misalignment, the AP-579 section 8.4.3.2 suggests applying a pure bending loading $\left(\sigma_{b}\right)$ into the model in the absence of the crack. The bending load to be applied is calculated 
using formulae from API-579 Table 8.9. Fig. 10 compares the stress distribution across the thickness of the pipeline derived from the finite element model to the distribution considered in the API-579.

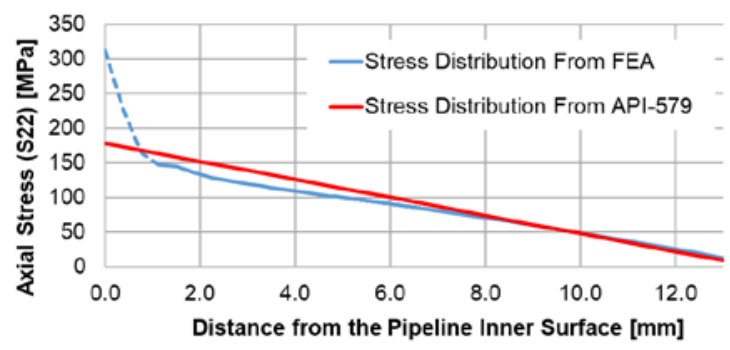

Fig. 10 Stress Field at Misalignment Location - No Crack

The high stresses observed in the first section of the stress distribution curve from the FEA (dashed line) are due to a stress concentration at the interface between the weld and the pipe. This stress concentration is - among other factors - at the origin of the crack initiation at this location.

It can be observed from this figure that, in the linear region, the stress distribution considered in the API-579 is more severe than the distribution observed in the finite element model.

\subsection{Variable Crack Depths}

Fig. 11 presents a comparison of the J-Integrals for various crack depth depending on the selected approach.

The methodology proposed by the API-579 in Annex C.5.7 has been validated by the industry and confirmed conservative by experiments for a majority of cases [19], [20]. The J-Integrals calculated from the FE model present a similar trend with the values calculated from the API for the considered case.

The conservative stress field considered in the thickness of the pipeline in the API-579 calculation (Fig. 10) tends to confirm the fact that results are in general larger when the API-579 is considered.

The API-579 also allows a higher order description of the stress field in the thickness of the pipeline using a fourth order polynomial to compute the Stress Intensity Factor. The use of this fourth order polynomial is very sensitive to the stress concentration observed at the inner surface of the pipe and shall be used with care. In the following, the entire ligament has been considered in the polynomial fit. Note that discarding the first few nodes of the ligament tend to lead to a slight reduction in the calculated J-Integrals.
The J-integral values from the "blunt" crack tip model are in general larger than the results from the "sharp" crack tip model.

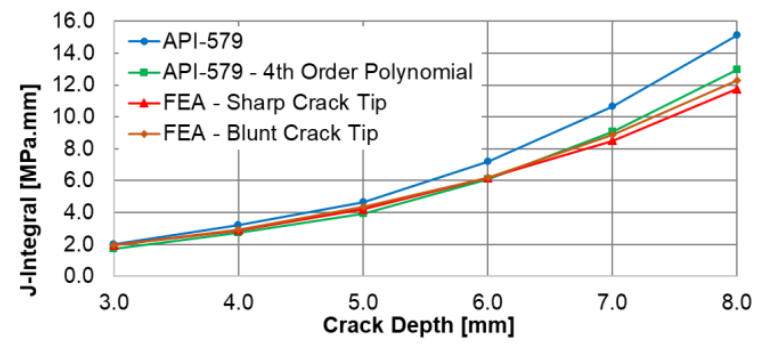

Fig. 11 J-Integral Results for Variable Crack Depths

\subsection{Variable Hi-Lo Misalignments}

The impact of the misalignment on the calculated J-Integral from the FE model and from the API-579 has also been assessed. The FE model capturing the elastic-plastic behaviour of the material has been selected. A sharp crack tip has been modelled using degenerated elements. A crack depth of $6 \mathrm{~mm}$ has been considered for this misalignment study.

Fig. 12 shows the result comparison for six misalignment values and the case of no misalignment.

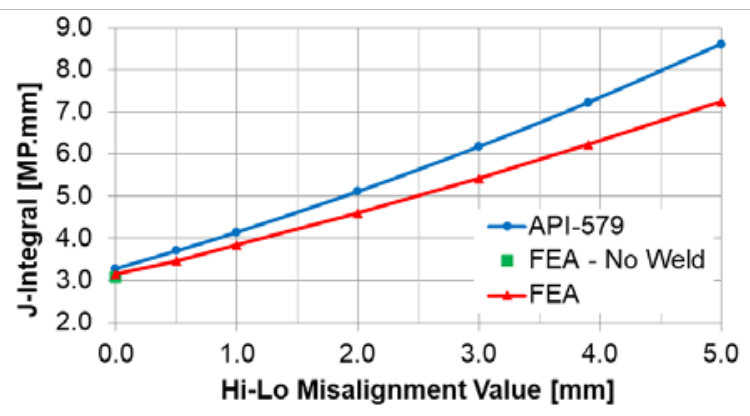

Fig. 12 Variable Hi-Lo Misalignments

Note: Modelling the weld material leads to an increase of $2.2 \%$ in the calculated $J$ Integral when no misalignment is present.

It can be observed that the J-Integrals calculated from the API-579 are larger than those calculated from the FE model. In a similar way as on Fig. 11, the relative difference between the two sets of values increases with the misalignment severity.

The presence of the weld in the pipeline model with no misalignment (additional welding material modelled) has a minor effect on the calculated JIntegral. 


\section{3D Pipeline Analysis - Strain Loading}

Following the preliminary two-dimensional assessment, a three-dimensional analysis has been performed for the case of the gas pipeline presented in Section 2.

A sharp crack tip modelling approach has been selected as it allows deriving relatively accurate results with a limited number of elements. A model including a Tie Constraint has also been chosen in order to refine sufficiently the mesh at the crack location while limiting the number of elements in the model and maintaining an acceptable element aspect ratio.

When only the internal pressure loading has been considered in the axisymmetric analysis, strain based loadings have been applied to the three-dimensional model to represent the typical loading induced by a landslide.

\subsection{Calculations and FAD}

The results from the 3D analysis have been analysed based on the calculated J-Integral. This allows determining the severity of the considered crack under the applied loading using the Failure Assessment Diagram (FAD).

Two parameters are computed for a given crack in order to assess its criticality: $\mathrm{Kr}$ and $\mathrm{Lr}$. $\mathrm{Kr}$ is the toughness ratio. It is the ratio of the stress intensity factor calculated for the considered crack and the material toughness. It depends on the material properties, on the crack dimensions and on the loading applied. $\mathrm{Lr}$ is the load ratio and is the ratio between a reference stress (see Annex D.5 of the API-579) of the considered load and the material yield stress. More details on the calculation of the $\mathrm{Kr}$ and $\mathrm{Lr}$ factors can be found in [1].

Fig. 13 shows a typical Failure Assessment Diagram. The "FAD Envelope" has two zones: the acceptable region for assessment points below this envelope and the unacceptable region for assessment points above. The equation of the FAD envelope depends on the considered material.

The location of the assessment point on the diagram shows the type of fracture susceptible to occur (i.e. Brittle Fracture, Plastic Collapse or Mixed Mode).

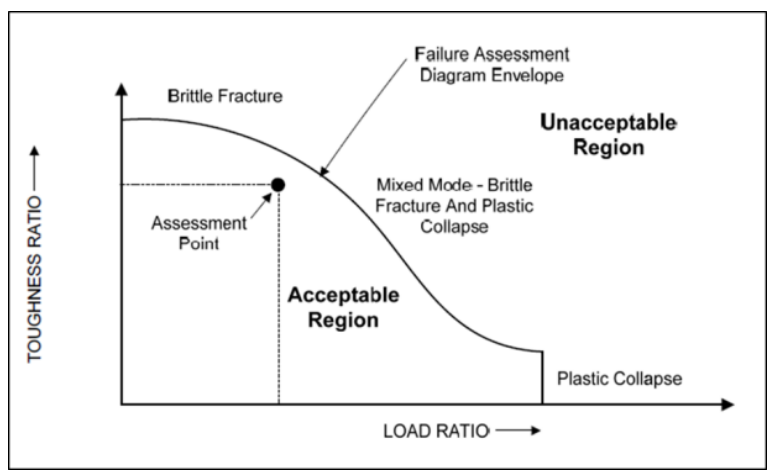

Fig. 13 Failure Assessment Diagram (FAD)

\subsection{The 3D Models Considered}

Fig. 14 shows a capture of the considered finite element model. In order to reduce the overall number of elements in the model and reduce the computation time, a 45deg sector of the pipeline has been conservatively considered. Symmetric boundary conditions are applied to each face of the sector. This models a pipeline with four cracks in the circumferential direction. The cracks are assumed sufficiently small for the four cracks in the section not to interact with each other. Consequently, the API-579 methodology (which considers only a single crack in the circumferential direction) can be applied.

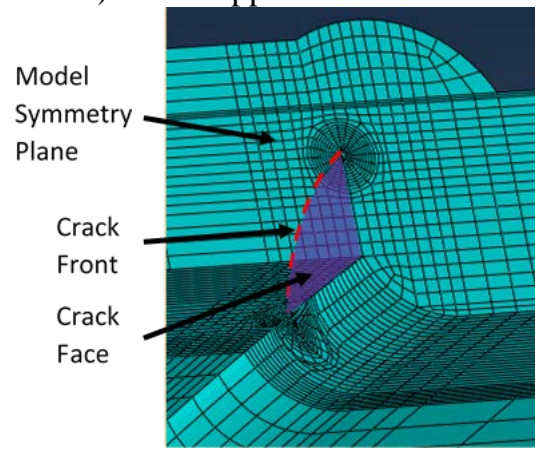

Fig. 14 3D FE Model

The dimensions of the analysed cracks have been presented in Table 3 below.

Table 3 Crack dimensions considered

\begin{tabular}{ccc}
\hline Crack & $\begin{array}{c}\text { Crack Depth } \\
\text { (a) [mm] }\end{array}$ & $\begin{array}{c}\text { Crack Length } \\
\text { (c) [mm] }\end{array}$ \\
\hline $\mathrm{a} 4 \mathrm{c} 8$ & 4.0 & 8.0 \\
\hline $\mathrm{a} 4 \mathrm{c} 20$ & 4.0 & 20.0 \\
\hline $\mathrm{a} 6 \mathrm{c} 10$ & 6.0 & 10.0 \\
\hline
\end{tabular}




\subsection{FEA vs API-579 - J-Integral along the Crack} Front

Results in terms of J-Integrals have been calculated from the FE model along the crack and compared to results derived from the API-579. As for the axisymmetric results, the $4^{\text {th }}$ order stress polynomial extracted from the FE model without crack has also been used in conjunction with API-579 for comparison purpose.

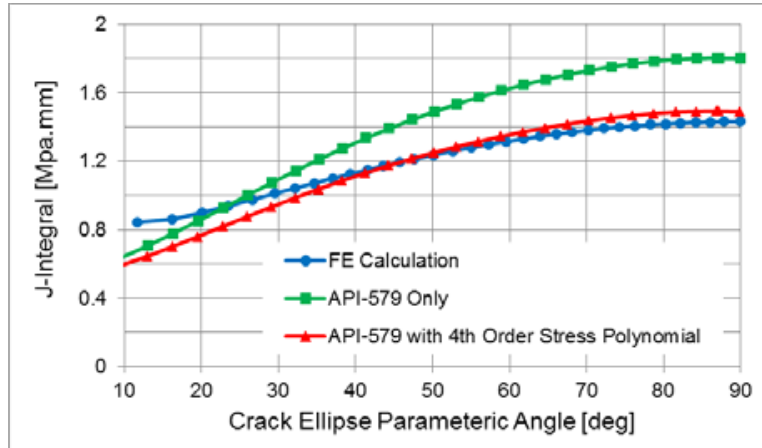

Fig. 15 API-579 vs FEA - J-Integral along Crack Front

Note: A crack ellipse parameter of 90deg corresponds to the deepest part of the crack front. See Fig. 6.

It can be observed from Fig. 15 that the results calculated using the "API-579 only" are in general larger than the results derived from the FE model. It is important to note that the model is deemed less accurate for ellipse parametric angles around zero due to the complexity of the interface between the crack and the weld at the misalignment location.

\subsection{Strain Based Loading}

Strain based loading has been applied to the modelled pipeline section in order to better represent an extreme loading condition due, for instance, to a landslide. The relation between the applied relative displacement and the average stress in the pipeline section is presented Fig 16. The Crack Opening for the various applied displacements is presented Fig. 17.

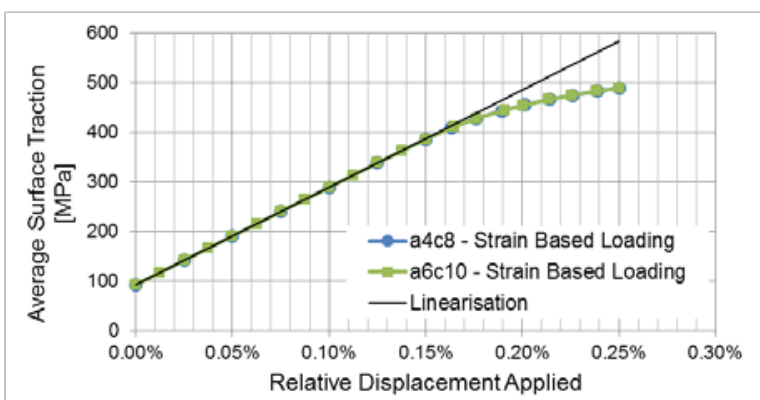

Fig. 16 Observed Stress under Strain Based Loading

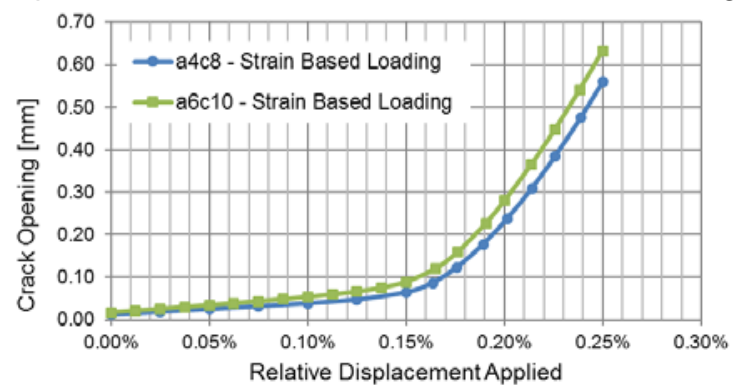

Fig. 17 Crack Opening under Strain Based Loading

Two sections can be identified from Fig. 16 and Fig. 17. For relative displacements between $0 \%$ and $0.16 \%$, the relation is linear. For larger displacements, the slope of the curve reduces progressively.

The linearization of the first section of the curves shows that the slope of this section is 196,929 MPa. This value is relatively close to the young modulus of the steel of the pipeline (200GPa). This small difference can be explained by the presence of the misalignment that induces a "structural" effect. For relative displacements larger than $0.16 \%$, the stress in the ligament is larger than the yield stress of the material and the plasticity of the material leads to a decrease in the slope of the stress / displacement curves.

The same two sections observed Fig. 176 are seen Fig. 17. For relative displacements larger than $0.16 \%$, the rapid increase of the Crack Opening is due to the ligament being fully plastic.

\subsection{Criticality Assessment - FAD Approach}

In order to assess the criticality of the considered cracks, an assessment using the FAD has been performed. Various assessment points corresponding to different applied displacements have been placed on the diagrams. J-integrals have been calculated for the crack ellipse parameters of 90deg (i.e. the deepest 
location of the crack font) and have been presented on a FAD Fig. 18.

The intersection between the FAD envelope and the curve defines when the loading becomes unacceptable for the considered crack.
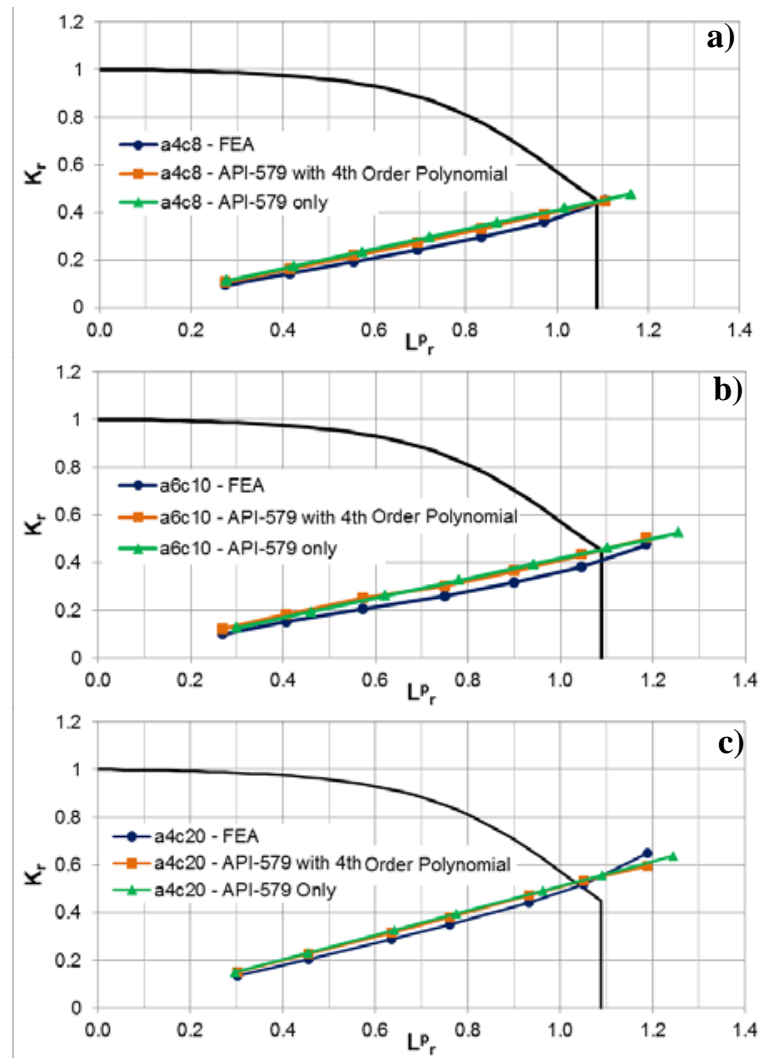

Fig. 188FADs for Increasing Strain Loadings

The limiting loadings for which the considered crack becomes unacceptable have been presented in Table 4 for the three calculation approaches.

It can be noticed from this table that the results from the FEA model and the API-579 with $4^{\text {th }}$ Order Polynomial are very close. Also, the crack length has little impact on the limiting loading compared to the crack depth. The limiting loadings derived from the API-579 only are smaller than those from the FEA.
Table 4. Limiting Loadings

\begin{tabular}{cccc}
\hline Calculation & \multicolumn{3}{c}{ Limiting Strain Loading } \\
\cline { 2 - 4 } Method & $\mathbf{a 4 c 8}$ & $\mathbf{a 4 c 2 0}$ & $\mathbf{a 6 c 1 0}$ \\
\hline FEA & $0.1477 \%$ & $0.1476 \%$ & $0.1360 \%$ \\
\hline $\begin{array}{c}\text { API-579 } \\
\text { with 4 } \\
\text { Order }\end{array}$ & $0.1478 \%$ & $0.1444 \%$ & $0.1321 \%$ \\
$\begin{array}{c}\text { Polynomial } \\
\text { API-579 } \\
\text { Only }\end{array}$ & $0.1375 \%$ & $0.1303 \%$ & $0.1225 \%$ \\
\hline
\end{tabular}

\subsection{Results Comparison in Terms of Remaining Life}

The gain achieved by the use of the FE method over the API-579 can be quantified in terms of life extension. For this purpose, the software package "Signal ${ }^{\text {TM }}$ Fitness-For-Service" has been used. The pipeline dimensions, the misalignment value and the crack dimensions are input in the software. An iterative method is used in order to determine the number of cycles before failure based on the API-579 methodology.

Fig. 9 shows on the FAD comparison of two equivalent cracks under the same loading. The assessment point determined based on the API-579 methodology has no fatigue life as it is located on the FAD envelope. The equivalent point determined using FEA is located inside of the acceptable zone of the FAD. Using the Paris law, the gain in terms of fatigue life can be determined. One pressure cycle per day has been considered conservatively.

The calculation performed with "SignalTM FitnessFor-Service" shows that more than 80 years (which is the expected life of a gas pipeline) of normal pipe operations are necessary for the assessment point calculated using the FE method to be considered unacceptable while deemed unacceptable using the "API-579 only" method.

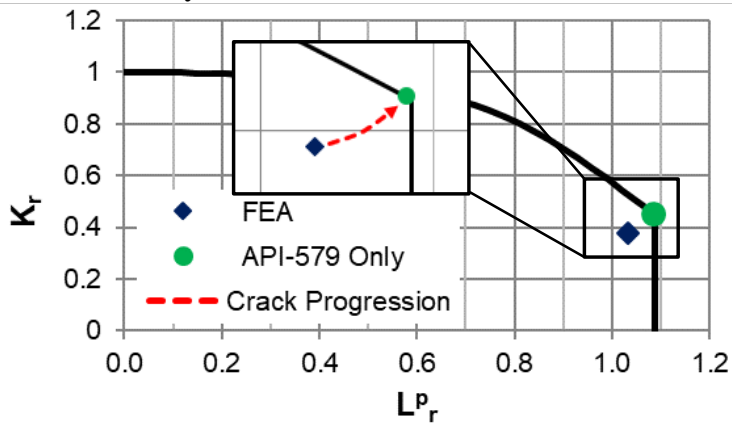

Fig. 19 Fatigue Life Comparison 
This shows that the API-579 approach may lead to conservative results, assuming a conservative stress field induced by the misalignment in the thickness of the pipeline ("API-579 only" method) and an elastic material. The use of the FE method allows, for the considered case, to accept a defect deemed unacceptable by the "API-579 only" approach. The stress field and the J-integrals calculated using the FEM shall be confirmed by testing.

\subsection{Remarks on the effect of residual stress}

It is well-known that the effect of the residual stress on the structural integrity and fatigue life can be significant in welded components. The region in and around the weld is particularly susceptible for failure due to tensile residual stress [21-22].

While computational techniques are now often employed to model residual stress generation, experimental validations are usually required due to complexity and varieties in welding techniques [23].

However, most welding methods generate 3D residual stress components with their 3D spatial variation and hence, it is very challenging to measure such comprehensive details with the existing residual stress measurement techniques [24-25]

As residual stress measurement techniques (particularly destructive ones) rely on the elastic relaxation, it can be appropriate to incorporate elastic residual stress into the developed models (such as the one in this study) as an initial stress field. In this way, the effect of residual stress can be directly observed.

\section{Conclusions}

The results presented in this paper show that the finite element method allows reproducing relatively accurate results from empirical relations such the Newman Raju formulation.

The two-dimensional analysis of the pipeline allowed better understanding the influence of the crack depth and misalignment value on the calculated $\mathrm{J}$ Integrals. This analysis also allowed comparing the results given from two modelling approaches (i.e. sharp and blunt crack tip) together with the results obtained from the API-579 standard.

The results show that for the considered case, the J-Integral calculated using the API-579 are larger than the J-Integrals calculated using the FE model. Among the two modelling approaches considered, modelling a blunt tip leads in general to slightly higher calculated J-Integrals.

The three-dimensional approach allowed developing a model closer to the reality of the problem. The stress field around the crack could be modelled allowing the calculation of J-Integrals along the semielliptical crack front. The criticality of the considered cracks has been assessed using a failure assessment diagram.

Finally, the analysis showed that for the case of an onshore pipeline having a crack in a misaligned circumferential weld, the FE method allows reducing the assumed conservatism of the API-579. When a $6 \mathrm{~mm}$ deep crack is considered unacceptable under normal operating conditions using the API-579 approach, the FE shows that this crack is acceptable (i.e. it has a remaining life over 80 years, assuming that the loading will not rise) preventing a costly repair.

It shall however be noted that meshing the crack region is time consuming for complex $3 \mathrm{D}$ geometries. The use of the "Tie Constrain" in ABAQUS allows reducing the meshing time and does not affect much the calculated results.

Crack assessments based on J-Integrals have limitations for elastic-plastic models as the stability of the calculated J-Integral contours can only be achieved for contours in the elastic region. The calculated JIntegrals shall be used with care and are not appropriate for large plastic deformations.

\section{References}

[1] Fitness-For-Service, API 579-1/ASME FFS-1, June 2007.

[2] Guide to methods for assessing the acceptability of flaws in metallic structures, BSI Standards Publication, BS 7910:2013.

[3] Y.M. Zhang, D.K. Yi, Z.M. Xiao, Z.H. Huang, S.B. Kumar (2013). Elastic-plastic fracture analyses for pipeline girth welds with 3D semielliptical surface cracks subjected to large plastic bending. International Journal of Pressure Vessels and Piping, vol. 105-106, pp. 90-102.

[4] Y.M. Zhang, Z.M. Xiao, W.G. Zhang, Z.H. Huang (2014), Strain-based CTOD estimation formulations for fracture assessment of offshore pipelines subjected to large plastic deformation, Ocean Engineering, vol. 91, pp. 64-72.

[5] Y.M. Zhang, Z.M. Xiao, W.G. Zhang (2013). On 3-D Crack problems in offshore pipeline 
with large plastic deformation, Theoretical and Applied Fracture Mechanics, vol. 67-68, pp. 2228.

[6] S.J. Lee, Y.C. Yoon, S.S. Hwang, W.Y. Cho, G. Zi (2011). Development of an evaluation method for the compressive bending plastic buckling capacity of pipeline steel tube based on strain based design in structural engineering and construction. Procedia Engineering, vol. 14, pp. 312-317.

[7] T. S. Chong, S. B. Kumar, M. On Lai, W. Lam Loh (2015). Fracture capacity of modern pipeline girth welds with 3D surface cracks under extreme operating conditions, Engineering Fracture Mechanics, vol. 146, pp. 139-160.

[8] D. Yi, Z. Min Xiao, S. Idapalati, S. B. Kumar (2012). Fracture analysis of girth welded pipeline with 3D embedded cracks subjected to biaxial loading conditions, Engineering Fracture Mechanics, vol. 96, pp. 570-587.

[9] I. Lotsberg (2009). Stress concentrations due to misalignment at butt welds in plated structures and at girth welds in tubulars, International Journal of Fatigue, vol. 31, pp. 1337-1345.

[10] H.G. Kunert A.A. Marquez, P. Fazzini, J.L. Otegui (2016), Failures and integrity of pipelines subjected to soil movements. Handbook of Materials Failure Analysis with Case Studies from the Oil and Gas Industry, chapter 5, pp. 105-122.

[11] F. Yuan, M. F. Randolph, L. Wang, L Zhao, Y. Tian (2014). Refined analytical models for pipelay on elasto-plastic seabed. Applied Ocean Research, vol. 48, pp. 292-300.

[12] W. Mohr (2003). Strain Based Design of Pipelines, TWI to U.S. Department of Interior, Minerals Management Service.

[13] Modelling Fracture and Failure with ABAQUS.

[14] ABAQUS 6.14 Theory Guide.

[15] Specification for Steel Pipes for Pipelines Common Requirements, TS-C4Gas-PIP0 v7.5, C4Gas, June 2011.

[16] CES EduPack (2016). The Cambridge Engineering Selector. Granta Design. Cambridge, UK

[17] J.R. Rice (1968). A Path Independent Integral and the Approximate Analysis of Strain Concentration by Notches and Cracks. Journal of Applied Mechanics, vol. 35(2), pp. 379-386.

[18] Newman, Raju (1981). An Empirical StressIntensity Factor Equation for Surface Crack, Engineering Fracture Mechanics, vol. 15 (1-2), pp.185-192.

[19] S. Cravero, C. Ruggieri (2006). Structural integrity analysis of axially cracked pipelines using conventional and constraint-modified failure assessment diagrams. International Journal of Pressure Vessels and Piping, vol. 83 (8) pp. 607-617.

[20] A. Hosseini et al, Experimental Testing and Evaluation of Crack Defects in Line Pipe. 8th International Pipeline Conference, Calgary, Alberta, Canada, September 27-October 1, 2010.

[21] M.E. Kartal M.. Turski, G. Johnson, S. Gungor, M.E. Fitzpatrick., P.J. Withers., L. Edwards (2006).Residual stress measurements in single and multi-pass groove weld specimens using neutron diffraction and the contour method. Materials Science Forum, vol. 524-525, pp. 671676.

[22] M.E. Kartal (2013). Analytical solutions for determining residual stresses in two-dimensional domains using the contour method. Proceedings of Royal Society A, 469, Article 20130367.

[23] M.E. Kartal, Y.H. Kang, A.M. Korsunsky, A.C.F. Cocks, J.P. Bouchard (2016). The influence of welding procedure and plate geometry on residual stresses in thick components. International Journal of Solids and Structures, vol. 80, pp. 420-429.

[24] M.E. Kartal, C.D.M. Liljedahl, S. Gungor, L. Edwards M.E. Fitzpatrick, (2008). Determination of the profile of the complete residual stress tensor in a VPPA weld using the multi-axial contour method. Acta Materialia., vol. 56 (16), pp. 4417-4428.

[25] M.E. Kartal, R. Kiwanuka, F.P.E. Dunne (2015). Determination of sub-surface stresses at inclusions in single crystal superalloy using HREBSD, crystal plasticity and inverse eigenstrain analysis. International Journal of Solids and Structures vol. 67, pp. 27-39. 